\title{
QUEUES AND RISK MODELS WITH SIMULTANEOUS ARRIVALS
}

\author{
E.S. BADILA, O.J. BOXMA, J.A.C. RESING, AND E.M.M. WINANDS
}

\begin{abstract}
We focus on a particular connection between queueing and risk models in a multi-dimensional setting. We first consider the joint workload process in a queueing model with parallel queues and simultaneous arrivals at the queues. For the case that the service times are ordered (from largest in the first queue to smallest in the last queue) we obtain the Laplace-Stieltjes transform of the joint stationary workload distribution. Using a multivariate duality argument between queueing and risk models, this also gives the Laplace transform of the survival probability of all books in a multivariate risk model with simultaneous claim arrivals and the same ordering between claim sizes.

Other features of the paper include a stochastic decomposition result for the workload vector, and an outline how the two-dimensional risk model with a general two-dimensional claim size distribution (hence without ordering of claim sizes) is related to a known Riemann boundary value problem.
\end{abstract}

\section{INTRODUCTION}

There are several connections between queueing and risk models. A classical result is that the ruin probability in the Cramér-Lundberg risk model, in which the arrival process of claims is a compound Poisson process, is related to the workload (or waiting time) in an $M / G / 1$ queue with the same compound Poisson input. More precisely, denoting by $\left(R_{t}\right)_{t \geq 0}$ the surplus process in the Cramér-Lundberg risk model, by $\tau$ the time of ruin of this process and by $\left(V_{t}\right)_{t \geq 0}$ the workload process in the corresponding $M / G / 1$ queue, one has $\mathbb{P}\left(\tau \leq t \mid R_{0}=u\right)=\mathbb{P}\left(V_{t}>u \mid V_{0}=0\right)$; in particular, the probability of ruin ever occurring when starting at $u$ equals the probability that the steady-state workload exceeds $u$. See, e.g., the nice geometric duality argument on page 46 of Asmussen and Albrecher [1, or Rolski et al. [22].

However, also other ruin-related performance measures have a counterpart in queueing theory. By interpreting the interarrival times of the claims as service times of the corresponding queue and the claim sizes as interarrival times of the queue, the standard Cramér-Lundberg model is translated into a $G / M / 1$ queue. The time to ruin in the Cramér-Lundberg model is now related to a busy period of the corresponding queue, the deficit at ruin to an idle period and the surplus just before ruin to the sojourn time of the last customer in a busy period (see Frostig [15] and Löpker and Perry [18]).

2010 Mathematics Subject Classification. 60K25; 91B30.

Key words and phrases. Queues with simultaneous arrivals, workload, stochastic decomposition, duality, multivariate risk model.

The authors gratefully acknowledge stimulating discussions with Yifan Xu (Binghamton University). Serban Badila is being supported by Project 613.001.017 of the Netherlands Organisation for Scientific Research (NWO). 
In this paper our focus is on a connection between queueing and risk models in a multi-dimensional setting. In particular, we look at the joint workload process in a queueing model with parallel queues and simultaneous arrivals at the queues. Under the condition that, with probability 1, the service times of the customers arriving at the same time at the different queues are ordered (i.e., the customer in queue 1 has the largest service time, the customer in queue 2 the second largest service time, and so on) we are able to find the Laplace-Stieltjes transform of the joint stationary workload distribution in the different queues. Using a multivariate duality argument between queueing and risk models, this immediately gives the Laplace transform of the survival (non-ruin) probabilities in a multivariate risk model with simultaneous claim arrivals (and the same ordering property for the claim sizes of the simultaneous claims at the different books in the model).

Queueing models with parallel queues and simultaneous arrivals are also often called fork-join queues. These models have many applications in computer-, communication- and production systems in which jobs are split among a number of different processors, communication channels or machines. Clearly, the queues in these models are dependent due to the simultaneous arrivals. In general this makes an exact analysis of the model very hard. Only in the case of two queues, exact results are available (see, e.g., Flatto and Hahn [14, Wright [25, Baccelli [4, De Klein [11 and Cohen [9). We will come back to some of these exact results in Section 6 of the paper. For the model with more than two servers no exact analytical results are available in the literature. In this case, bounds and approximations for several performance measures have been developed, see e.g. [5, 20, 21.

Multivariate risk models with simultaneous claim arrivals have several applications in the area of ruin theory. One example is provided by reinsurance models in which, whenever a claim arrives, several insurance companies pay a part of the claim. Another example would be a large insurance company with multiple lines of business, where correlated claims arrive at the various business lines. Albeit in a different area of risk management, analysis of the dependence between the stochastic asset processes of several counter parties is also one of the most challenging aspects in the field of credit risk. Especially, in a two-dimensional setting one has to study the joint asset process of an obligor and a guarantor in credit default swaps.

Avram, Palmowski and Pistorius [2, 3] have studied the joint ruin problem for the special case of two insurance companies that divide between them both claims and premia in some specific proportions. In particular, they derive the double Laplace transform with respect to the two initial reserves of the survival probabilities of the two companies. Proportional claims are a special case of our ordered claims, and we show in Section 4 that their survival result indeed is a special case of our Formula (8). One of the key observations in [2, 3] is that, due to the fact that the companies divide the claims in some specific proportions, the two-dimensional ruin problem may be viewed as a one-dimensional crossing problem over a piecewise linear barrier. Badescu, Cheung and Rabehasaina [6] have extended the two-dimensional model of [2, 3] by allowing, next to the arrivals of claims for which the two insurers divide the claim in some specific proportions, also extra arrivals of claims which are fully paid by one of the insurers (e.g., insurer 1). They show that under some conditions also in this model the previously mentioned reduction to a 1-dimensional problem still holds. However, in [6] the authors do not consider the double Laplace transform with respect to the two initial reserves of the survival probabilities of the 
two companies (their main focus is on the Laplace transform of the time until ruin of at least one insurer).

The remainder of the paper is organized as follows: In Section 2 we present our model in detail and we provide the multivariate duality argument. This duality argument allows a translation between results for the queueing model and results for the multivariate risk model. Section 3 is dedicated to the analysis of the 2-dimensional queueing model with ordered service times. After introducing the assumptions, we derive the Laplace-Stieltjes transform of the joint stationary workloads in the two queues and present a decomposition theorem for the stationary workload in the two queues. In Section 5 we extend the results of Section 3 to the $K$-dimensional queueing model. Section 4 is dedicated to relations to other models. We present connections with tandem and priority queues, but also with a reinsurance problem with proportional claim sizes. In Section 6 we discuss the case of a general two-dimensional service time (or claim size) distribution. We indicate that the two-dimensional workload problem has been solved in the queueing literature. The solution is very complicated; our ordered service times case is a degenerate case, but a case which has the advantage of a much more explicit solution which offers more probabilistic insight - and a case that can be generalized to higher dimensions. Finally, Section 7 outlines possible further research directions.

Among the main contributions of our paper, we mention an explicit result for the transform of the joint workload (respectively, of the joint survival probability) and its extension to the $K$-dimensional model. In addition, we mention the workload decomposition result. It seems to be new in this setting, although similar results - under the assumption of independent inputs - were obtained for parallel queues (cf. [17]). From a more abstract perspective, another contribution of our paper is that it strengthens the links between queueing and risk models, pointing out that certain results and methods in the literature (and in the present paper) for queues with simultaneous arrivals are of immediate use in the risk setting, and vice versa.

\section{Multivariate Duality}

We consider a $K$-dimensional risk process in which claims arrive simultaneously in the $\mathrm{K}$ branches, according to a Poisson process with rate $\lambda$. The claim sizes in the $K$ books are independent, identically distributed random vectors $\left(B_{n}^{(1)}, \ldots, B_{n}^{(K)}\right)$, $n \geq 1$. In the sequel we denote with $\left(B^{(1)}, \ldots, B^{(K)}\right)$ a random vector with the same distribution as $\left(B_{1}^{(1)}, \ldots, B_{1}^{(K)}\right)$.

For the $\mathrm{n}^{\text {th }}$ arriving claim vector, denote by $A_{n}$ the time elapsed since the arrival of the previous claim vector, so that the $A_{n}$ are independent and have an identical exponential distribution with parameter $\lambda$.

Let $R_{t}^{(i)}, i=1, \ldots, K$ be $K$ risk reserve processes with initial capitals $u_{i}$, premium rates $c^{(i)}$ and the same arrival instants $\sigma_{n}, n \geq 1$. We have $A_{n}=\sigma_{n}-\sigma_{n-1}$ and $\sigma_{0}=0$ (no delay). Then

$$
R_{t}^{(i)}=u_{i}+\sum_{j=1}^{n(t)}\left(c^{(i)} A_{j}-B_{j}^{(i)}\right)+c^{(i)}\left(t-\sigma_{n(t)}\right),
$$

where $n(t)$ is the number of arrivals before $t$. Let $\tau^{(i)}\left(u_{i}\right)=\inf \left\{t>0: R_{t}^{(i)}<0\right\}$ be the times to ruin. 
In connection with the ruin process, we consider $K$ parallel $M / G / 1$ queues with simultaneous (coupled) arrivals and correlated service requirements. As in the ruin setting, $A_{n}$ are the interarrival times of customers in the $K$ queues and the vector $\left(B^{(1)}, \ldots, B^{(K)}\right)$ denotes the generic service requirements. The speed of server $i$ is denoted by $c^{(i)}$, meaning that server $i$ handles $c^{(i)}$ units of work per time unit, $i=1, \ldots, K$.

Furthermore we denote by $\rho_{i}:=\lambda \mathbb{E}\left(B^{(i)}\right)$ the load of queue $i, i=1, \ldots, K$ and we assume that $\rho_{i}<c^{(i)}$, to ensure that all queues can handle the offered traffic. These conditions imply positive safety loading in the ruin setting.

From the queueing perspective, let $\left(V_{t}^{(1)}, \ldots, V_{t}^{(K)}\right)$ be the workload vector at time $t$ in the system or, if we consider the $n^{t h}$ arrival epoch, this is the workload $\left(V_{n}^{(1)}, \ldots, V_{n}^{(K)}\right)$ seen by the customers of the $n^{t h}$ batch arrival. Remark that $V_{n}^{(i)}=c^{(i)} W_{n}^{(i)}$, with $W_{n}^{(i)}$ the waiting time of the $n^{t h}$ arrival in queue $i$. Under the stability conditions above, the vectors $\left(V_{t}^{(1)}, \ldots, V_{t}^{(K)}\right)$ and $\left(V_{n}^{(1)}, \ldots, V_{n}^{(K)}\right)$ converge in distribution to the steady-state joint workload at arbitrary epochs and at arrival epochs, respectively. Due to the PASTA property these vectors are equal. Similarly, the vector $\left(W_{n}^{(1)}, \ldots, W_{n}^{(K)}\right)$ converges in distribution to the steady state waiting time. We denote the Laplace-Stieltjes transform (LST) of the steady-state workload vector:

$$
\psi\left(s_{1}, s_{2}, \ldots, s_{K}\right):=\mathbb{E}\left(e^{-s_{1} V^{(1)}-s_{2} V^{(2)}-\ldots-s_{K} V^{(K)}}\right) .
$$

For the multidimensional ruin process defined in (1), consider a dual workload process with $V_{N}^{(i)}, i=1, \ldots, K$ the workload seen upon arrival by the $N^{t h}$ customer in $K$ initially empty queues with the time reverted arrival process (the arrival epochs are the same for all the systems):

$$
\sigma_{n}^{*}=\sigma_{N-n+1}, \quad\left(A_{n}^{*}=A_{N-n+1}\right), \quad n=1, \ldots, N
$$

service time of customer $n$ at queue $i: B_{n}^{*(i)}=B_{N-n+1}^{(i)}, n=1, \ldots, N$ (time reverted service time) (cf. [1]).

The following lemma shows that the well-known duality result (cf. [1, p. 46) between the Cramér-Lundberg model and the $M / G / 1$ queue can be extended to the multivariate risk model and the queueing model with simultaneous arrivals. Here the connection is between the various possibilities to be ruined (i.e we may have ruin in all books or precisely in one, at least in one, etc.) The results below are presented for the case $K=2$, but can be directly extended to the general case.

Lemma 1. The following identities hold:

(a) $\left\{V_{N}^{(1)}>u_{1} \wedge V_{N}^{(2)}>u_{2}\right\}=\left\{\tau^{(1)}\left(u_{1}\right) \leq \sigma_{N} \wedge \tau^{(2)}\left(u_{2}\right) \leq \sigma_{N}\right\}$

(b) $\left\{V_{N}^{(1)} \leq u_{1} \wedge V_{N}^{(2)} \leq u_{2}\right\}=\left\{\tau^{(1)}\left(u_{1}\right)>\sigma_{N} \wedge \tau^{(2)}\left(u_{2}\right)>\sigma_{N}\right\}$

(c) $\left\{V_{N}^{(1)}>u_{1} \wedge V_{N}^{(2)} \leq u_{2}\right\}=\left\{\tau^{(1)}\left(u_{1}\right) \leq \sigma_{N} \wedge \tau^{(2)}\left(u_{2}\right)>\sigma_{N}\right\}$

(d) $\left\{V_{N}^{(1)} \leq u_{1} \wedge V_{N}^{(2)}>u_{2}\right\}=\left\{\tau^{(1)}\left(u_{1}\right)>\sigma_{N} \wedge \tau^{(2)}\left(u_{2}\right) \leq \sigma_{N}\right\}$

The above relations are path-wise identities.

Proof. The following identities hold for the cylinder sets:

$$
\left\{V_{N}^{(i)}>u_{i}\right\}=\left\{\tau^{(i)}\left(u_{i}\right) \leq \sigma_{N}\right\}
$$


This follows directly from Asmussen and Albrecher ([1, page 46) for the 1-dimensional problem, and is a special case of the duality in Siegmund [23].

If we intersect the above identities, we obtain $(a)$. (b) follows by intersecting their complements, and $(c)$ and $(d)$ by subtracting $(b)$ and $(a)$ respectively, from the complements of the above cylinder sets. This concludes the proof.

If we let $N \rightarrow \infty$ in (b) of Lemma 1, we obtain the infinite horizon joint survival probability

$$
\lim _{N \rightarrow \infty} \mathbb{P}\left(V_{N}^{(1)} \leq u_{1} \wedge V_{N}^{(2)} \leq u_{2}\right)=\mathbb{P}\left(\tau^{(1)}\left(u_{1}\right)=\infty \wedge \tau^{(2)}\left(u_{2}\right)=\infty\right) .
$$

Denote the righthand side by $\xi\left(u_{1}, u_{2}\right)$. This is the joint survival function, for initial capital $\left(u_{1}, u_{2}\right)$. By PASTA, we can replace the steady state workload at arrival epochs with the steady state workload at arbitrary epochs in (2).

Let

$$
\xi_{*}(s, t):=\int e^{-s x_{1}-t x_{2}} \xi\left(x_{1}, x_{2}\right) d x_{1} d x_{2}
$$

be the Laplace transform (LT) of the joint survival function. Via (2), this is also the LT of the c.d.f. of the joint workload in steady state. By a simple integration by parts, we have the following relation with the LST of the workload:

$$
\xi_{*}(s, t)=\frac{1}{s t} \psi(s, t) .
$$

\section{ThE ANALYSIS OF THE TWO-DIMENSIONAL PROBLEM}

In this section we derive the transform of the joint steady state workload process of the two-dimensional queueing model with simultaneous arrivals, as introduced in Section 2. We also present a probabilistic interpretation of the quantities involved in the formula of the joint workload. The results are of immediate relevance for the corresponding insurance problem, via the duality outlined in the previous section.

Before we start with the analysis, we make the following simplifying assumption. Assumption 1. All premium rates, respectively all service speeds, are 1, viz., $c^{(1)}=\cdots=c^{(K)}=1$.

The following observation shows that this assumption is not restrictive. If we divide all terms in the righthand side of 1 by $c^{(i)}$, we arrive at a new risk model with initial capital $u_{i} / c^{(i)}$ and claim size $B^{(i)} / c^{(i)}$ and unit premium rates. Similarly, in the corresponding queueing model the service times at queue $i$ are also divided by $c^{(i)}$ and the service speeds are equal to 1 . This will not change the $n^{\text {th }}$ waiting time $W_{n}^{(i)}$ at queue $i$, but the workload $V_{n}^{(i)}$ at the $n^{\text {th }}$ arrival epoch is divided by $c^{(i)}$. Also the times to ruin are preserved, hence the identities in Lemma 1 from the previous section remain unchanged.

The LST of the joint service time/claim size vector is denoted by

$$
\phi(s, t):=\mathbb{E}\left(e^{-s B^{(1)}-t B^{(2)}}\right) .
$$

Our key assumption is the following:

Assumption 2. $\mathbb{P}\left(B^{(1)} \geq B^{(2)}\right)=1$. In view of the above discussion, in the case of speeds $c^{(i)}$ our assumption would be $\mathbb{P}\left(B^{(1)} / c^{(1)} \geq B^{(2)} / c^{(2)}\right)=1$. 
Remark 1. This model allows for a dedicated Poisson arrival stream into queue 1. Merging this separate arrival process with the simultaneous arrival process at queue 1 , the distribution of $B^{(2)}$ will have an atom in 0 , which is the probability that a dedicated Poisson arrival happens instead of a simultaneous one (see Badescu et al. [6] for a reinsurance model with both dedicated and simultaneous arrivals).

We are interested in the joint stationary distribution of the amount of work in the two queues

$$
\psi(s, t):=\mathbb{E}\left(e^{-s V^{(1)}-t V^{(2)}}\right) .
$$

This can be obtained in the following way. Consider the amount of work in queue $i$ just before the arrival of customer $n$. We have the following recursion for the random variables $\left(V_{n}^{(1)}, V_{n}^{(2)}\right), n=1,2, \ldots$

$$
\left(V_{n+1}^{(1)}, V_{n+1}^{(2)}\right)=\left(\max \left(V_{n}^{(1)}+B_{n}^{(1)}-A_{n}, 0\right), \max \left(V_{n}^{(2)}+B_{n}^{(2)}-A_{n}, 0\right)\right) .
$$

Or, for the LST

$$
\psi_{n}(s, t)=\mathbb{E}\left(e^{-s V_{n}^{(1)}-t V_{n}^{(2)}}\right), \quad n=1,2, \ldots,
$$

this gives after straightforward calculations

$$
\begin{aligned}
\psi_{n+1}(s, t) & =\frac{\lambda}{\lambda-s-t}\left(\phi(s, t) \psi_{n}(s, t)-\phi(s, \lambda-s) \psi_{n}(s, \lambda-s)\right) \\
& +\frac{\lambda}{\lambda-s}\left(\phi(s, \lambda-s) \psi_{n}(s, \lambda-s)-\phi(\lambda, 0) \psi_{n}(\lambda, 0)\right) \\
& +\phi(\lambda, 0) \psi_{n}(\lambda, 0) .
\end{aligned}
$$

Under the stability condition $\rho_{1}<1, \psi(s, t):=\lim _{n \rightarrow \infty} \psi_{n}(s, t)$ exists and

$$
\begin{aligned}
\left(1-\frac{\lambda \phi(s, t)}{\lambda-s-t}\right) \psi(s, t) & =\left(\frac{\lambda}{\lambda-s}-\frac{\lambda}{\lambda-s-t}\right) \phi(s, \lambda-s) \psi(s, \lambda-s) \\
& +\left(1-\frac{\lambda}{\lambda-s}\right) \phi(\lambda, 0) \psi(\lambda, 0) .
\end{aligned}
$$

If we let $A$ denote a generic interarrival time, then due to the PASTA property,

$$
\phi(\lambda, 0) \psi(\lambda, 0)=\mathbb{P}\left(V^{(1)}+B^{(1)} \leq A\right)=\mathbb{P}\left(V^{(1)}=0\right)=1-\rho_{1} .
$$

This is the probability that queue 1 is empty at an arbitrary time instant.

On the regularity domains of $\psi(s, t)$ and $\phi(s, t)$ : We remark that, because of the dependence $\mathbb{P}\left(B^{(1)} \geq B^{(2)}\right)=1$, we can rewrite the transform of the joint service times as:

$$
\phi(s, t)=\mathbb{E} e^{-s\left(B^{(1)}-B^{(2)}\right)-(s+t) B^{(2)}}=: \tilde{\phi}(s, s+t),
$$

and this function is always regular in $\mathcal{R} e s>0, \mathcal{R} e(s+t)>0$. If we consider $\left(B^{(1)}, B^{(2)}\right)$ subject to $B^{(1)} \geq B^{(2)}$ a.s., $\phi(s, t)$ may not be regular beyond this domain. More precisely, if $B^{(2)}$ has a heavy-tailed distribution, this implies that $B^{(1)}$ is also heavy tailed because of the dependence structure. In this case $\phi(s, t)$ cannot be extended beyond $\mathcal{R} e s \geq 0, \mathcal{R} e(s+t) \geq 0$. Similar considerations hold for $\psi(s, t)$ because we must also have $\mathbb{P}\left(V^{(1)} \geq V^{(2)}\right)=1$.

By Lemma 1 in the Appendix, $\forall s$ with $\mathcal{R} e s>0$, there is a unique $t(s)$, well defined and analytic in Re $s>0$, such that $\lambda \phi(s, t(s))=\lambda-s-t(s)$. Hence $(s, t(s))$ is a zero of $\left(1-\frac{\lambda \phi(s, t)}{\lambda-s-t}\right)$ in $\sqrt[5]{ }$, which is in the regularity domain of $\psi(s, t)$. Then the righthand side of (5) is also zero, i.e.

$$
\lambda t(s) \phi(s, \lambda-s) \psi(s, \lambda-s)=-s(\lambda-t(s)-s) \phi(\lambda, 0) \psi(\lambda, 0) .
$$


If we substitute this in (5) and use (6), we obtain

$$
\psi(s, t)=\left(1-\rho_{1}\right) \frac{s}{s+t-\lambda(1-\phi(s, t))} \cdot \frac{t(s)-t}{t(s)} .
$$

The interpretation of the Rouché zero $t(s)$. Assume that a customer that starts a busy period $B P^{(2)}$ in queue 2 demands work $x$ in queue 2 and work $x+y$ in queue 1. During the service time of this customer in the second queue, there are Poisson $(\lambda x)$ arriving customers, each one of these generating an i.i.d. busy sub-period with the same distribution as $B P^{(2)}$ in queue 2. So if we denote with $U$ the extra work in the first queue, at the end of a busy period in the second queue, and with $U^{*}(s)$ its Laplace-Stieltjes transform, we have the identity:

$$
U^{*}(s)=\int_{x=0}^{\infty} \int_{y=0}^{\infty} e^{-s y} \sum_{k=0}^{\infty} \frac{(\lambda x)^{k}}{k !} e^{-\lambda x}\left[U^{*}(s)\right]^{k} d \mathbb{P}\left(B^{(1)}-B^{(2)} \leq y, B^{(2)} \leq x\right)
$$

The powers of $U^{*}(s)$ correspond to the extra work contributions at the end of the busy sub-periods started during the service time of the first customer in the busy period $B P^{(2)}$. We can rewrite the above identity as:

$$
U^{*}(s)=\tilde{\phi}\left(s, \lambda\left[1-U^{*}(s)\right]\right)=\phi\left(s, \lambda\left[1-U^{*}(s)\right]-s\right) .
$$

Comparing this with the equation in Lemma 1 in terms of $\tilde{\phi}(s, s+t)$, we have:

$$
\left\{\begin{array}{l}
\lambda \tilde{\phi}(s, s+t(s))=\lambda-(s+t(s)) \\
\lambda \tilde{\phi}\left(s, \lambda\left[1-U^{*}(s)\right]\right)=\lambda U^{*}(s) .
\end{array}\right.
$$

We may assume w.l.o.g. that $\mathbb{P}\left(B^{(1)}>B^{(2)}\right)>0$, otherwise the two queues are a.s. identical, which is not interesting. Then it follows that the real part of $\lambda\left(1-U^{*}(s)\right)$ is positive, and we must have $s+t(s)=\lambda\left(1-U^{*}(s)\right)$ because the solution obtained in Lemma 1 is unique in the region $\mathcal{R} e(s+t)>0$. We have thus proved:

Proposition 1. The relation between $t(s)$ and the transform of the extra workload in queue 1 at the end of a busy period in the shortest queue is

$$
\lambda U^{*}(s)=\lambda-(s+t(s))
$$

The transform of the joint workload in the two systems becomes

$$
\psi(s, t)=\left(1-\rho_{1}\right) \frac{s+t-\lambda\left(1-U^{*}(s)\right)}{s+t-\lambda(1-\phi(s, t))} \cdot \frac{s}{s-\lambda\left(1-U^{*}(s)\right)} .
$$

The workload decomposition. Based on Proposition 1 , we show that the steadystate workload decomposes into an independent sum of a modified workload and an additional term, which represents the steady-state workload in a classical M/G/1 queue.

We start the joint workload process and let it run until the end of each busy period in the queue with the smallest workload. At this random time instant, we remove the extra content in queue 1, which has the largest workload of the two. Let 
us denote this modified joint workload process as $\left(\tilde{V}^{(1)}, V^{(2)}\right)$. Then at the arrival instants of customers in the two queues, the recurrence relation holds:

$$
\left(\tilde{V}_{n+1}^{(1)}, V_{n+1}^{(2)}\right)= \begin{cases}\left(\tilde{V}_{n}^{(1)}+B_{n}^{(1)}-A_{n}, V_{n}^{(2)}+B_{n}^{(2)}-A_{n}\right), & \text { if } A_{n}<V_{n}^{(2)}+B_{n}^{(2)} \\ (0,0), & \text { if } A_{n} \geq V_{n}^{(2)}+B_{n}^{(2)} .\end{cases}
$$

Remark that marginally, the shortest queue evolves unchanged.

If we have ergodicity then in steady state, the above recurrence becomes:

$$
\left(\tilde{V}^{(1)}, V^{(2)}\right) \stackrel{d}{=} \begin{cases}\left(\tilde{V}^{(1)}+B^{(1)}-A, V^{(2)}+B^{(2)}-A\right), & \text { if } A<V^{(2)}+B^{(2)} \\ (0,0), & \text { if } A \geq V^{(2)}+B^{(2)}\end{cases}
$$

Here and in the following, $\stackrel{d}{=}$ denotes equality in distribution. If we rewrite this in terms of LST's, we obtain the following functional equation for $\tilde{\psi}(s, t):=$ $\mathbb{E} e^{-s \tilde{V}^{(1)}-t V^{(2)}}$ :

$$
\left(1-\frac{\lambda \phi(s, t)}{\lambda-s-t}\right) \tilde{\psi}(s, t)=\left(1-\rho_{2}\right)-\frac{\lambda}{\lambda-s-t} \tilde{\psi}(s, \lambda-s) \phi(s, \lambda-s),
$$

where $1-\rho_{2}=\mathbb{P}\left(V^{(2)}=0\right)$.

Now follows a similar analysis as for $\psi(s, t)$. We already know from the Rouché problem that $t(s)$ from Lemma 1 is a zero of $\left(1-\frac{\lambda \phi(s, t)}{\lambda-s-t}\right)$. We also have $\tilde{V}^{(1)} \geq V^{(2)}$ a.s. (even if we take out the extra workload at the largest queue at the end of each busy period, $\tilde{V}^{(1)}$ is still at least as large as $V^{(2)}$ in the long run), therefore $(s, t(s))$ is in the regularity domain of $\tilde{\psi}(s, t)$ and therefore, at the point $(s, t(s))$, the right-hand side of the above identity is equal to zero:

$$
\tilde{\psi}(s, \lambda-s) \phi(s, \lambda-s)=\left(1-\rho_{2}\right) \frac{\lambda-s-t(s)}{\lambda} .
$$

Substituting back in the original identity, yields:

$$
\tilde{\psi}(s, t)=\left(1-\rho_{2}\right) \frac{s+t-\lambda(1-\phi(s, t(s)))}{s+t-\lambda(1-\phi(s, t))} .
$$

This is a 2-dimensional Pollaczek-Khinchine type of representation. From an analytic point of view, the role of the numerator is to cancel the unique pole of the denominator in the region $\mathcal{R e}(s+t)>0$.

Substitute $t(s)$ from Proposition 1 and $\tilde{\psi}$ from (11) into (8):

$$
\psi(s, t)=\frac{1-\rho_{1}}{1-\rho_{2}} \frac{s}{s-\lambda\left[1-U^{*}(s)\right]} \tilde{\psi}(s, t) .
$$

We can now state the main result:

Theorem 1 (Work decomposition). In steady state, we have the following representation of the joint workload at the two queues as an independent sum:

$$
\left(V^{(1)}, V^{(2)}\right) \stackrel{d}{=}\left(\tilde{V}^{(1)}, V^{(2)}\right)+\left(V^{(1), 1}, 0\right),
$$

where $V^{(1), 1}$ is the workload in an independent, virtual $M / G / 1$ queue with arrival rate $\lambda$ and service requirements distributed as $U$, the extra workload at the end of a busy period $B P^{(2)}$ in the shortest queue. 
Proof. It suffices to remark that the factor

$$
\frac{1-\rho_{1}}{1-\rho_{2}} \frac{s}{s-\lambda\left[1-U^{*}(s)\right]}=\mathbb{E} e^{-s V^{(1), 1}}
$$

in 12 is the Pollaczek-Khinchine formula for the transform of the workload in the virtual $\mathrm{M} / \mathrm{G} / 1$ queue with service time distribution $U$. This virtual queue is obtained by contracting the busy periods in the initial shortest queue, so that an arrival in the virtual queue happens at the end of this busy period and the interarrival time is then the idle period in the initial queue, and so is exponentially distributed.

To see that indeed $\frac{1-\rho_{1}}{1-\rho_{2}}$ is the atom of $V^{(1), 1}$ at 0 , differentiate the identity for $U^{*}(s)$ in $(9)$ :

$$
\mathbb{E}(U)=-\frac{d}{d s} \phi\left(s, \lambda\left(1-U^{*}(s)-s\right)\right)_{\mid s=0}=\mathbb{E}\left(B^{(1)}-B^{(2)}\right)+\lambda \mathbb{E} B^{(1)} \mathbb{E}(U)
$$

so that $1-\lambda \mathbb{E}(U)=\frac{1-\rho_{1}}{1-\rho_{2}}$.

\section{Relation With Other MOdels}

In this section we point out how the results of the previous section are related to results for a risk model with proportional reinsurance, a particular tandem fluid model and with a particular priority queue. We start by showing that (8) generalizes a result obtained in [3, for the risk setting.

The case of proportional reinsurance. In $[3]$ the joint reserve process $\left(R^{(1)}, R^{(2)}\right)$ is of the form: $R^{(i)}(t)=u_{i}+c^{(i)} t / \delta_{i}-S(t)$. Here $S(t)$ is a common Compound Poisson input process with generic claim sizes $\sigma$ and $c^{(i)}$ are the premium rates. The claims are being divided in fixed proportions $\delta_{i}$, respectively.

To bring this closer to our setting in Section 3, normalize the income rates: i.e. we consider $\left(\frac{1}{p_{1}} R^{(1)}, \frac{1}{p_{2}} R^{(2)}\right)$ with $p_{i}=\frac{c^{(i)}}{\delta_{i}}$. The assumption in [3] is that $p_{1}>p_{2}$, which means that, in our notation, the claim sizes are $B^{(1)}:=\frac{1}{p_{1}} \sigma<\frac{1}{p_{2}} \sigma=: B^{(2)}$. Remark that the inequality between the $B^{(i)}$ 's is reversed here (which means the role of the arguments in our transforms is interchanged, especially the Rouché zero).

Let us recall the main formula in [3] (Formula (23)):

$$
\psi_{* R^{(1)}, R^{(2)}}(p, q)=\frac{\kappa_{2}(0+)^{\prime}}{p\left(\kappa_{1}(p+q)-q\left(p_{1}-p_{2}\right)\right)} \frac{q+p-q^{+}\left(q\left(p_{1}-p_{2}\right)\right)}{q-q^{+}\left(q\left(p_{1}-p_{2}\right)\right)} .
$$

The relation between the ruin times of $\left(R^{(1)}, R^{(2)}\right)$ and $\left(\frac{1}{p_{1}} R^{(1)}, \frac{1}{p_{2}} R^{(2)}\right)$ is

$$
\tau_{\frac{1}{p_{1}} R^{(1)}, \frac{1}{p_{2}} R^{(2)}}\left(u_{1}, u_{2}\right)=\tau_{R^{(1)}, R^{(2)}}\left(p_{1} u_{1}, p_{2} u_{2}\right) .
$$

Hence the relation to the LT coordinates used in (3) is $s=p_{1} p, t=p_{2} q$. From this, the relation between the LT of the survival functions becomes after a change of variables:

$$
\psi_{* \frac{1}{p_{1}} R^{(1)}, \frac{1}{p_{2}} R^{(2)}}(s, t)=\frac{1}{p_{1} p_{2}} \psi_{* R^{(1)}, R^{(2)}}(p, q) .
$$

- $\kappa_{i}(\alpha)$ is the Laplace exponent of the Compound Poisson process with drift $p_{i}$ per unit time. This means

$$
\kappa_{i}(\alpha)=p_{i} \alpha-\lambda\left(1-\mathbb{E} e^{-\alpha \sigma}\right) .
$$


Because of the linear dependence between the $B^{(i)}$ 's, their LST has the form $\mathbb{E} e^{-s B^{(1)}-t B^{(2)}}=\phi(s, t)=: \phi_{B^{(1)}}\left(s+\frac{p_{1}}{p_{2}} t\right)$.

- $q^{+}(q)$ is the largest root of the equation $\kappa_{1}(\alpha)=q$. Then $q^{+}\left(q\left(p_{1}-p_{2}\right)\right)$ solves:

$$
p_{1} \alpha-\lambda\left(1-\mathbb{E} e^{-\alpha p_{1} B^{(1)}}\right)=q\left(p_{1}-p_{2}\right) .
$$

Remark that if we set $\alpha=p+q$, the above becomes:

$$
p_{1} p+p_{2} q-\lambda\left(1-\phi_{B^{(1)}}\left(p_{1} p+p_{1} q\right)\right)=0,
$$

or, written in the $(s, t)$-coordinates, this becomes the equation in Lemma 1 (with $s$ and $t$ interchanged). Hence the relation between the zeroes in the two notations is: $s(t)=p_{1}(\alpha-q)=p_{1}\left[q^{+}\left(q\left(p_{1}-p_{2}\right)\right)-q\right]$.

The constant $\kappa(0+)^{\prime}=p_{2}-\lambda \mathbb{E} B^{(2)}=p_{2}\left(1-\rho_{2}\right)$ is the probability that the queueing system is empty in steady state (now the second queue has a higher workload).

In conclusion, (13) written via (14) and (3) in the $(s, t)$ coordinates becomes Formula (8):

$$
\psi(t, s)=\frac{s\left(1-\rho_{2}\right)}{s+t-\lambda\left(1-\phi_{B^{(1)}}\left(s+\frac{p_{1}}{p_{2}} t\right)\right)} \cdot \frac{s-s(t)}{-s(t)},
$$

with the arguments $s$ and $t$ interchanged.

Relation with work on tandem fluid queues. We now show that the workload model with ordered service times is equivalent with a particular tandem fluid queue. That is a model of two queues in series, in which the outflow from the first queue is a fluid, i.e., there is continuous outflow when the server is working (instead of customers leaving one by one). Such tandem fluid queues have been studied by various authors, see in particular [17]. Consider the following two-station tandem fluid network with independent compound Poisson input at the two stations (with arrival rate $\lambda_{i}$ and Laplace-Stieltjes transform of the service times $\left.B_{i}^{*}(\cdot), i=1,2\right)$. Then Theorem 4.1 of Kella [17] gives the Laplace-Stieltjes transform of the steadystate fluid levels $W_{1}$ and $W_{2}$ in the two nodes:

$$
\psi_{W}\left(\alpha_{1}, \alpha_{2}\right)=\mathbb{E}\left(e^{-\alpha_{1} W_{1}-\alpha_{2} W_{2}}\right)=\frac{\left(1-\rho_{1}-\rho_{2}\right) \alpha_{2}}{\phi_{1}\left(\alpha_{1}\right)-\phi_{1}\left(\hat{\eta}_{2}\left(\alpha_{2}\right)\right)} \cdot \frac{\alpha_{1}-\hat{\eta}_{2}\left(\alpha_{2}\right)}{\alpha_{2}-\hat{\eta}_{2}\left(\alpha_{2}\right)},
$$

with

- $\rho_{i}=\lambda_{i} \mathbb{E}\left(B_{i}\right)$,

- $\phi_{1}\left(\alpha_{1}\right)=\alpha_{1}-\eta_{1}\left(\alpha_{1}\right)$,

- $\eta_{i}\left(\alpha_{i}\right)=\lambda_{i}\left(1-B_{i}^{*}\left(\alpha_{i}\right)\right)$,

- $\hat{\eta}_{2}\left(\alpha_{2}\right)$ the solution of $\phi_{1}\left(\hat{\eta}_{2}\left(\alpha_{2}\right)\right)=\eta_{2}\left(\alpha_{2}\right)$.

Alternatively, the last relation can also be formulated as: $\hat{\eta}_{2}\left(\alpha_{2}\right)$ is the solution of

$$
\lambda_{1} B_{1}^{*}\left(\hat{\eta}_{2}\left(\alpha_{2}\right)\right)+\lambda_{2} B_{2}^{*}\left(\alpha_{2}\right)=\lambda_{1}+\lambda_{2}-\hat{\eta}_{2}\left(\alpha_{2}\right) .
$$

This system is related to our model with arrival rate $\lambda=\lambda_{1}+\lambda_{2}$ and LaplaceStieltjes transform of service requirements

$$
\phi(s, t)=\frac{\lambda_{1}}{\lambda_{1}+\lambda_{2}} B_{1}^{*}(s+t)+\frac{\lambda_{2}}{\lambda_{1}+\lambda_{2}} B_{2}^{*}(s) .
$$

The corresponding notation is: $B_{1} \stackrel{d}{=} B^{(2)}$ and $B_{2} \stackrel{d}{=} B^{(1)}-B^{(2)}$. Here $W_{1}$ in the tandem model corresponds to the workload in the smallest queue in our model and 


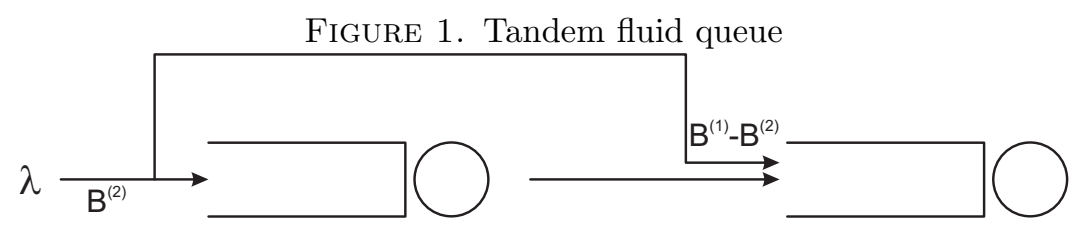

$W_{1}+W_{2}$ in the tandem model corresponds to the workload in the largest queue in our model. So we have

$$
\begin{aligned}
\psi(s, t) & =\mathbb{E}\left(e^{-s V_{1}-t V_{2}}\right)=\mathbb{E}\left(e^{-s\left(W_{1}+W_{2}\right)-t W_{1}}\right)=\psi_{W}(s+t, s) \\
& =\frac{\left(1-\rho_{1}-\rho_{2}\right) s}{s+t-\lambda_{1}\left(1-B_{1}^{*}(s+t)\right)-\lambda_{2}\left(1-B_{2}^{*}(s)\right)} \cdot \frac{s+t-\hat{\eta}_{2}(s)}{s-\hat{\eta}_{2}(s)} .
\end{aligned}
$$

Now remark that

- The total traffic offered to the largest queue is $\rho_{1}+\rho_{2}$, so indeed the factor $1-\rho_{1}-\rho_{2}$ in [17] corresponds to the factor $1-\rho_{1}$ in (8);

- $\lambda(1-\phi(s, t))=\lambda_{1}\left(1-B_{1}^{*}(s+t)\right)+\lambda_{2}\left(1-B_{2}^{*}(s)\right)$;

- $\lambda \phi(s, t(s))=\lambda_{1} B_{1}^{*}(s+t(s))+\lambda_{2} B_{2}^{*}(s)=\lambda_{1}+\lambda_{2}-(s+t(s))$, so indeed $\hat{\eta}_{2}(s)$ corresponds to our $s+t(s)$.

We conclude that (8) coincides with Theorem 4.1 of [17] in the case of independent compound Poisson input. Kella's result is more general in the sense that he has Lévy input instead of compound Poisson input. Our result is more general in the sense that we have dependent compound Poisson input.

Relation with work on priority queues. As was already noticed in Kella [17, but also in several other places in the literature, the tandem fluid network described above is also related to a priority queue with preemptive resume priorities. Hence the same holds for our workload model. Consider the following model with two types of customers where customers of type- $i$ arrive according to a Poisson process with rate $\lambda_{i}$ having service times with Laplace-Stieltjes transform $B_{i}^{*}(\cdot), i=1,2$. Assume furthermore that customers of type- 1 have preemptive resume priority over customers of type-2. If we denote by $Y_{1}$ and $Y_{2}$ the steady-state workloads in the two queues, then $Y_{1}$ and $Y_{2}$ are related to $W_{1}$ and $W_{2}$ in the tandem fluid network. The Laplace-Stieltjes transform of the steady-state workloads in the two queues satisfies

$$
\psi_{Y}(s, t)=\mathbb{E}\left(e^{-s Y_{1}-t Y_{2}}\right)=\mathbb{E}\left(e^{-s W_{1}-t W_{2}}\right)=\mathbb{E}\left(e^{-s V_{2}-t\left(V_{1}-V_{2}\right)}\right)=\psi_{V}(t, s-t)
$$

where again in our model we have to take arrival rate $\lambda=\lambda_{1}+\lambda_{2}$ and LaplaceStieltjes transform of service requirements

$$
\phi(s, t)=\frac{\lambda_{1}}{\lambda_{1}+\lambda_{2}} B_{1}^{*}(s+t)+\frac{\lambda_{2}}{\lambda_{1}+\lambda_{2}} B_{2}^{*}(s) .
$$

We conclude that (8) also gives the Laplace-Stieltjes transform of a priority queue. Again our result is more general in the sense that we have dependent compound Poisson input (i.e., we can have arrivals of customers who have both low and high priority work). 


\section{The $K$-Dimensional PROBlem}

. In this section we consider the $K$-queue system with simultaneous arrivals. We give the transform for the steady-state joint workload and we show that the decomposition in Theorem 1 extends to this case if we preserve the ordering between the service requirements/claim sizes. We use an iterative argument and for this purpose, the decomposition in Section 3 will be the starting point; the iteration step is essentially done with the help of Lemma 2 below as a work conservation identity.

. We thus consider $K$ parallel $M / G / 1$ queues, numbered 1 to $K$, respectively, with simultaneous (coupled) arrivals and correlated service requirements. The arrival process is again a Poisson process with rate $\lambda$. The service requirements of successive customers at the $\mathrm{K}$ queues are independent, identically distributed random vectors $\left(B_{n}^{(1)}, \ldots, B_{n}^{(K)}\right), n \geq 1$. Let $\left(B^{(1)}, \ldots, B^{(K)}\right)$ be a generic random vector with the same distribution as $\left(B_{1}^{(1)}, \ldots, B_{1}^{(K)}\right)$. The LST of the service time/claim size vector is denoted by

$$
\phi\left(s_{1}, \ldots, s_{K}\right):=\mathbb{E}\left(e^{-s_{1} B^{(1)}-\cdots-s_{K} B^{(K)}}\right) .
$$

The essential assumption in the model extends Assumption 2 for the 2-dimensional problem:

$$
\mathbb{P}\left(B^{(1)} \geq B^{(2)} \geq \cdots \geq B^{(K)}\right)=1 .
$$

Furthermore we denote by $\rho_{i}:=\lambda \mathbb{E} B^{(i)}, i=1, \ldots, K$, the load of queue $i$ and we assume that $\rho_{1}<1$ (hence $\rho_{i}<1, \forall i$ ), to assure that all queues can handle the offered work.

Remark 2. Like in the two-dimensional case (cf. Remark 1), this model allows for a separate Poisson arrival stream into queue 1. Merging this separate arrival process with the simultaneous arrival process, the distribution of $\left(B^{(2)}, \ldots, B^{(K)}\right)$ will have an atom in $(0, \ldots, 0)$, which is the probability that a dedicated Poisson arrival happens instead of a simultaneous one.

Similarly, the model allows for simultaneous arrivals at the first $j$ queues only. This can be achieved by letting the distribution of $\left(B^{(j+1)}, \ldots, B^{(K)}\right)$ have an atom at $(0, \ldots, 0)$.

The Laplace-Stieltjes transform of $\left(V^{(1)}, \ldots, V^{(K)}\right)$. Denote the Laplace-Stieltjes transform of the service time/claim size vector by

$$
\phi\left(s_{1}, \ldots, s_{K}\right):=\mathbb{E}\left(e^{-s_{1} B^{(1)}-\cdots-s_{K} B^{(K)}}\right) .
$$

We have the $K$-dimensional Lindley recursion for the random variables $\left(V_{n}^{(1)}, \ldots, V_{n}^{(K)}\right)$ :

$\left(V_{n+1}^{(1)}, \ldots, V_{n+1}^{(K)}\right)=\left(\max \left(V_{n}^{(1)}+B_{n}^{(1)}-A_{n}, 0\right), \ldots, \max \left(V_{n}^{(K)}+B_{n}^{(K)}-A_{n}, 0\right)\right) n \geq 1$.

For the LST:

$$
\psi_{n}\left(s_{1}, \ldots, s_{K}\right)=\mathbb{E}\left(e^{-s_{1} V_{n}^{(1)}-\cdots-s_{K} V_{n}^{(K)}}\right), \quad n \geq 1,
$$

the Lindley recursion gives after straightforward calculations:

$$
\begin{aligned}
\psi_{n+1}\left(s_{1}, \ldots, s_{K}\right) & =\sum_{j=1}^{K} \frac{\lambda}{\lambda-\sum_{i=1}^{j} s_{i}}\left[\phi^{(j)}\left(s_{1}, \ldots, s_{j}\right) \psi_{n}^{(j)}\left(s_{1}, \ldots, s_{j}\right)\right. \\
& \left.-\phi^{(j-1)}\left(s_{1}, \ldots, s_{j-1}\right) \psi_{n}^{(j-1)}\left(s_{1}, \ldots, s_{j-1}\right)\right]+\phi^{(0)} \psi_{n}^{(0)}
\end{aligned}
$$


where we used the following notation for simplicity: $\psi_{n}^{(K)}\left(s_{1}, \ldots, s_{K}\right):=\psi_{n}\left(s_{1}, \ldots, s_{K}\right)$ and $\psi_{n}^{(0)}:=\psi_{n}(\lambda, 0, \ldots, 0)$, and

$$
\psi_{n}^{(j)}\left(s_{1}, \ldots, s_{j}\right):=\psi_{n}(s_{1}, \ldots, s_{j}, \lambda-\sum_{i=1}^{j} s_{i}, \underbrace{0, \ldots, 0}_{K-j-1 \text { arguments }}), \text { for } 1 \leq j \leq K-1 .
$$

$\phi^{(j)}\left(s_{1}, \ldots, s_{j}\right)$ is analogously defined for $j=0, \ldots, K$. By taking $n \rightarrow \infty$ in 16 , we obtain for $\psi\left(s_{1}, \ldots, s_{K}\right):=\lim _{n \rightarrow \infty} \psi_{n}\left(s_{1}, \ldots, s_{K}\right)$,

$$
\begin{aligned}
\left(1-\frac{\lambda \phi\left(s_{1}, \ldots, s_{K}\right)}{\lambda-\sum_{i=1}^{K} s_{i}}\right) \psi\left(s_{1}, \ldots, s_{K}\right) & =\sum_{j=0}^{K-1}\left(\frac{\lambda}{\lambda-\sum_{i=1}^{j} s_{i}}-\frac{\lambda}{\lambda-\sum_{i=1}^{j+1} s_{i}}\right) \\
& \cdot \phi^{(j)}\left(s_{1}, \ldots, s_{j}\right) \psi^{(j)}\left(s_{1}, \ldots, s_{j}\right),
\end{aligned}
$$

with $\psi^{(j)}:=\lim _{n \rightarrow \infty} \psi_{n}^{(j)}$; and $\phi^{(0)} \psi^{(0)}=\mathbb{P}\left(V^{(1)}+B^{(1)} \leq A\right)=1-\rho_{1}$.

Formula (17) has a simple recursive structure, and we can rewrite it as:

$$
\begin{aligned}
& \left(1-\frac{\lambda \phi\left(s_{1}, \ldots, s_{K}\right)}{\lambda-\sum_{i=1}^{K} s_{i}}\right) \psi\left(s_{1}, \ldots, s_{K}\right)=\left(\frac{\lambda}{\lambda-\sum_{i=1}^{K-1} s_{i}}-\frac{\lambda}{\lambda-\sum_{i=1}^{K} s_{i}}\right) \phi^{(K-1)}\left(s_{1}, \ldots, s_{K-1}\right) . \\
& (18) \quad \psi^{(K-1)}\left(s_{1}, \ldots, s_{K-1}\right)+\left(1-\frac{\lambda \phi\left(s_{1}, \ldots, s_{K-1}, 0\right)}{\lambda-\sum_{i=1}^{K-1} s_{i}}\right) \psi\left(s_{1}, \ldots, s_{K-1}, 0\right) .
\end{aligned}
$$

Denote by $C_{j}:=\left(1-\frac{\lambda \phi\left(s_{1}, \ldots, s_{j}, 0, \ldots, 0\right)}{\lambda-\sum_{i=1}^{j} s_{i}}\right) \psi\left(s_{1}, \ldots, s_{j}, 0, \ldots, 0\right)$, and remark that $\psi\left(s_{1}, \ldots, s_{j}, 0, \ldots, 0\right)$ is the transform of the workload in the $j$-dimensional system obtained by ignoring the last $(K-j)$ queues, $j=1, \ldots, K$.

Proposition 2. The LST of the steady-state workload in the $K \geq 3$ systems is given by:

$$
\psi\left(s_{1}, \ldots, s_{K}\right)=\frac{\left(1-\rho_{K}\right)\left(S_{K}-s_{K}\right)}{\sum_{i=1}^{K} s_{i}-\lambda\left(1-\phi\left(s_{1}, \ldots, s_{K}\right)\right)} \prod_{j=2}^{K-1} \frac{1-\rho_{j}}{1-\rho_{j+1}} \frac{S_{j}-s_{j}}{S_{j+1}} \cdot \frac{1-\rho_{1}}{1-\rho_{2}} \frac{s_{1}}{S_{2}},
$$

with $S_{j}=S_{j}\left(s_{1}, \ldots, s_{j-1}\right)$ the unique solution of the equation

$$
\lambda \phi\left(s_{1}, \ldots, s_{j}, 0, \ldots, 0\right)=\lambda-\sum_{i=1}^{j} s_{i}
$$

with $\mathcal{R} e\left(s_{1}+\cdots+s_{j-1}+S_{j}\left(s_{1}, \ldots, s_{j-1}\right)\right)>0$, for all $j=2, \ldots, K$.

Proof. The key remark is that $s_{K}$ is not among the arguments of the functions $\psi^{(j)}$ that appear in the righthand side of (17).

From Lemma 1 applied to $s=s_{1}+\cdots+s_{K-1}$ and $t=s_{K}$, there exists a unique solution $S_{K}=S_{K}\left(s_{1}, \ldots, s_{K-1}\right)$ of the equation

$$
\lambda \phi\left(s_{1}, \ldots, s_{K}\right)=\lambda-\sum_{i=1}^{K} s_{i}
$$

such that $S_{K}\left(s_{1}, \ldots, s_{K-1}\right)+\sum_{i=1}^{K-1} s_{i}$ has positive real part. Hence the hypersurface given by $S_{K}=S_{K}\left(s_{1}, \ldots, s_{K-1}\right)$ is contained in the regularity domain of $\psi\left(s_{1}, \ldots, s_{K}\right)$, and then the righthand side of 18 must be zero. This gives the following relation for $\psi^{(K-1)}\left(s_{1}, \ldots, s_{K-1}\right)$ : 


$$
\phi^{(K-1)}\left(s_{1}, \ldots, s_{K-1}\right) \psi^{(K-1)}\left(s_{1}, \ldots, s_{K-1}\right)=\frac{\left(\lambda-\sum_{i=1}^{K-1} s_{i}\right) \phi\left(s_{1}, \ldots, s_{K-1}, S_{K}\right)}{S_{K}} C_{K-1} .
$$

By substituting back into Equation (18), we obtain the recursion

$$
C_{K}=\frac{\lambda-\sum_{i=1}^{K-1} s_{i}}{\lambda-\sum_{i=1}^{K} s_{i}} \cdot \frac{S_{K}-s_{K}}{S_{K}} C_{K-1}
$$

with initial condition $C_{2}=-\left(1-\rho_{1}\right) \frac{s_{1}}{\lambda-s_{1}-s_{2}} \frac{S_{2}-s_{2}}{S_{2}}$, which follows from 8). From this, the formula in $(19)$ is obtained, after rearranging the factors.

Interpretation of the Rouché root. It is worthwhile to change the coordinates: $\left(s_{1}, s_{2}, \ldots, s_{K}\right) \rightarrow\left(s_{1}, s_{2}, \ldots, s_{K-1}, \sum_{i=1}^{K} s_{i}\right)$. We can rewrite

$$
\phi\left(s_{1}, \ldots, s_{K}\right)=\mathbb{E} e^{-s_{1}\left(B^{(1)}-B^{(K)}\right)-\ldots-s_{K-1}\left(B^{(K-1)}-B^{(K)}\right)-\left(\sum_{i=1}^{K} s_{i}\right) B^{(K)}}
$$

Let us denote it by $\tilde{\phi}\left(s_{1}, \ldots, s_{K-1}, \sum_{i=1}^{K} s_{i}\right)$. This is the transform of the extra service time (relative to the shortest queue) in the first $K-1$ queues, together with the shortest one. It turns out there is a connection between $s_{K}\left(s_{1}, \ldots, s_{K-1}\right)$ and the joint extra work in systems 1 to $K-1$ at the end of a busy period in system $K$. Let us denote this extra work by $\left(U_{1}, U_{2}, \ldots, U_{K-1}\right)$, with LST $U_{K}^{*}\left(s_{1}, \ldots, s_{K-1}\right)$, and let $F\left(x_{1}, x_{2}, \ldots, x_{K}\right)$ be the multivariate c.d.f. of $\left(B^{(1)}-B^{(K)}, \ldots, B^{(K-1)}-B^{(K)}, B^{(K)}\right)$. Then by a similar argument as the one leading to formula $(9), U_{K}^{*}\left(s_{1}, \ldots, s_{K-1}\right)$ satisfies the identity

$U_{K}^{*}\left(s_{1}, \ldots, s_{K-1}\right)=\int e^{-\sum_{i=1}^{K-1} s_{i} x_{i}} \sum_{n=0}^{\infty} \frac{\left(\lambda x_{K}\right)^{n}}{n !} e^{-\lambda x_{K}}\left[U_{K}^{*}\left(s_{1}, \ldots, s_{K-1}\right)\right]^{n} F\left(d x_{1} \ldots d x_{K}\right)$

$$
=\tilde{\phi}\left(s_{1}, \ldots, s_{K-1}, \lambda\left[1-U_{K}^{*}\left(s_{1}, \ldots, s_{K-1}\right)\right]\right) .
$$

Comparing this with the identity for the Rouché root

$$
\lambda-\left(s_{1}+\cdots+s_{K-1}+S_{K}\right)=\lambda \tilde{\phi}\left(s_{1}, \ldots, s_{K-1}, s_{1}+\cdots+s_{K-1}+S_{K}\right),
$$

gives the relation analogous to 10 in Proposition 1

$$
\lambda U_{K}^{*}\left(s_{1}, \ldots, s_{K-1}\right)=\lambda-\left(s_{1}+\cdots+s_{K-1}+S_{K}\right),
$$

which follows because the Rouché root is unique.

Let us fix our attention on the case $K=3$ for the moment. Then identity becomes

$$
\psi\left(s_{1}, s_{2}, s_{3}\right)=\frac{\left(1-\rho_{3}\right)\left(S_{3}-s_{3}\right)}{s_{1}+s_{2}+s_{3}-\lambda\left[1-\phi\left(s_{1}, s_{2}, s_{3}\right)\right]} \cdot \frac{1-\rho_{2}}{1-\rho_{3}} \frac{S_{2}-s_{2}}{S_{3}} \cdot \frac{1-\rho_{1}}{1-\rho_{2}} \frac{s_{1}}{S_{2}} .
$$



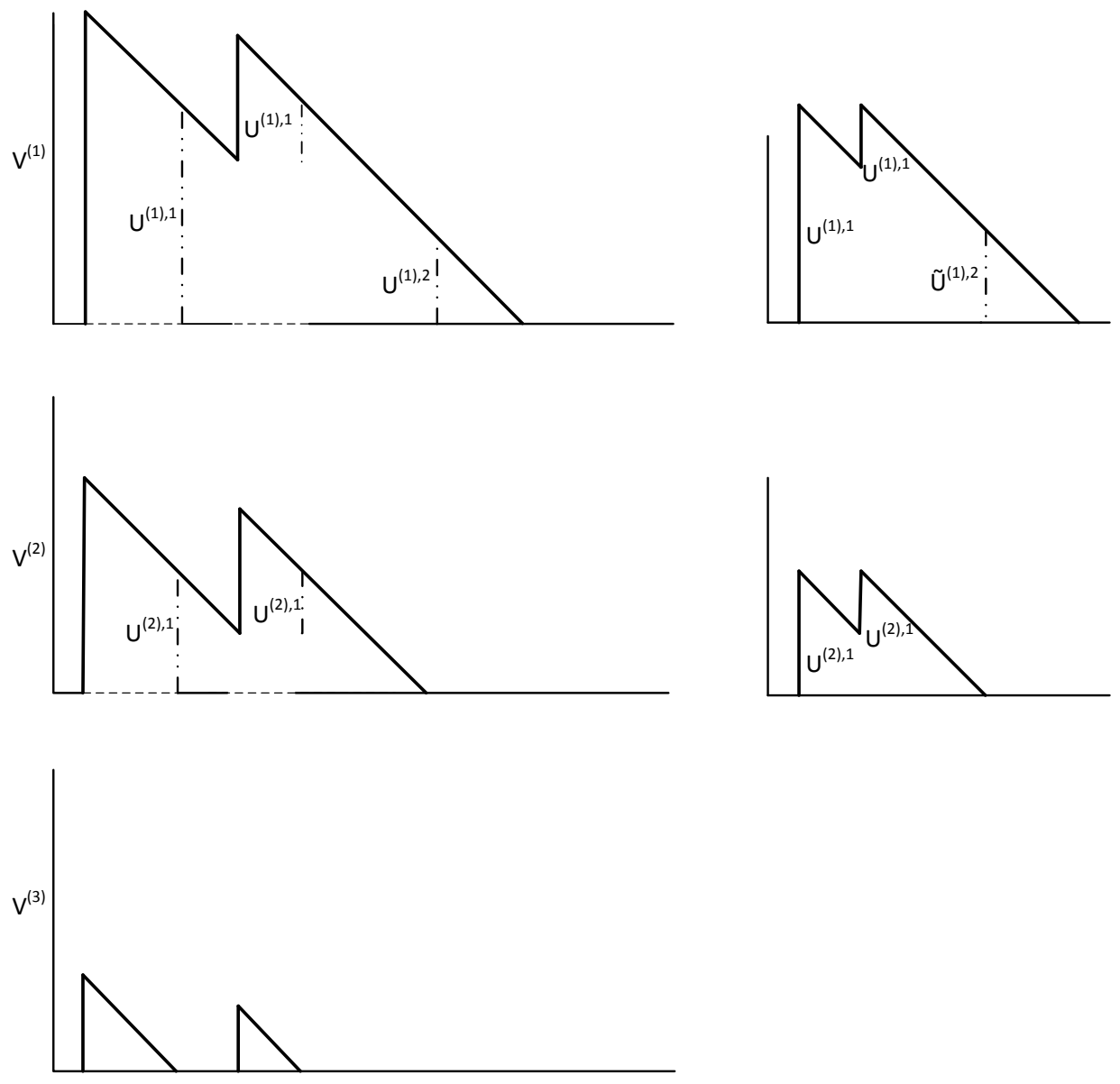

Figure 2. Work in the original system (left) and in the virtual system (right)

Work conservation. We would like to give a probabilistic interpretation of 22, In order to achieve this, we start by considering the joint extra work in queues 1 and 2 at the end of a busy period in queue 3. This has LST $U_{3}^{*}\left(s_{1}, s_{2}\right)$ as input in a 2-dimensional system with simultaneous Poisson arrivals, which is obtained by contracting the busy cycles in queue 3 . We call this the 2 -dimensional virtual system. Remark that the inter-arrival times in the virtual system are precisely the idle periods in queue 3 .

For this construction, the key observation is that the steady-state extra work in the virtual queue 1 at the end of the busy period in the virtual queue 2 is the same as the extra work in the initial queue 1 at the end of the busy period in the original queue 2. In analytic form, let $\tilde{U}_{2}^{*}\left(s_{1}\right)$ be the LST of the extra work in the virtual system and $U_{2}^{*}\left(s_{1}\right)$ be the LST of the extra work in the original system, see Figure 2 . 


\section{Lemma 2.}

$$
\tilde{U}_{2}^{*}\left(s_{1}\right)=U_{2}^{*}\left(s_{1}\right) .
$$

Proof. We begin by remarking that the extra work $\left(U^{(1), 1}, U^{(2), 1}\right)$ in the first 2 queues at the end of a busy period in queue 3 satisfies the a.s. inequality $U^{(1), 1} \geq$ $U^{(2), 1}$. Since this is the input in the virtual system, from Proposition $1 . \tilde{U}_{2}^{*}\left(s_{1}\right)$ satisfies the identity $(9)$ with $U_{3}^{*}\left(s_{1}, s_{2}\right)$ instead of $\phi\left(s_{1}, s_{2}\right)$ :

$$
U_{3}^{*}\left(s_{1}, \lambda\left[1-\tilde{U}_{2}^{*}\left(s_{1}\right)\right]-s_{1}\right)=\tilde{U}_{2}^{*}\left(s_{1}\right) .
$$

At the same time, via $20 p, U_{3}^{*}\left(s_{1}, s_{2}\right)$ satisfies

$$
\phi\left(s_{1}, s_{2}, \lambda\left(1-U_{3}^{*}\left(s_{1}, s_{2}\right)\right)-s_{1}-s_{2}\right)=U_{3}^{*}\left(s_{1}, s_{2}\right) .
$$

If we substitute this fixed point identity in $(23)$ above, we have

$$
\phi\left(s_{1}, \lambda\left(1-\tilde{U}_{2}^{*}\left(s_{1}\right)\right)-s_{1}, 0\right)=\tilde{U}_{2}^{*}\left(s_{1}\right) .
$$

On the other hand, this is also the identity $(9)$ satisfied by $U_{2}^{*}\left(s_{1}\right)$, in the 2dimensional system obtained by ignoring the last queue. Hence, from the uniqueness result in Lemma 1. $\tilde{U}_{2}^{*}\left(s_{1}\right)=U_{2}^{*}\left(s_{1}\right)$ (See Figure 2). This completes the proof.

We can rewrite (19) using (21):

$$
\begin{aligned}
\psi\left(s_{1}, s_{2}, s_{3}\right) & =\left(1-\rho_{3}\right) \frac{s_{1}+s_{2}+s_{3}-\lambda\left(1-U_{3}^{*}\left(s_{1}, s_{2}\right)\right)}{s_{1}+s_{2}+s_{3}-\lambda\left(1-\phi\left(s_{1}, s_{2}, s_{3}\right)\right)} \\
& \cdot \frac{1-\rho_{2}}{1-\rho_{3}} \frac{s_{1}+s_{2}-\lambda\left(1-U_{2}^{*}\left(s_{1}\right)\right)}{s_{1}+s_{2}-\lambda\left(1-U_{3}^{*}\left(s_{1}, s_{2}\right)\right)} \cdot \frac{1-\rho_{1}}{1-\rho_{2}} \frac{s_{1}}{s_{1}-\lambda\left(1-\tilde{U}_{2}^{*}\left(s_{1}\right)\right)}
\end{aligned}
$$

Remark that the atom $\frac{1-\rho_{1}}{1-\rho_{2}}$ above is the conditional probability that queue 1 is empty, given that queue 2 is empty; and similarly for $\frac{1-\rho_{2}}{1-\rho_{3}}$. In addition, the last factor in 24) is the Pollaczek-Khinchine representation for an M/G/1 queue with service times having $\operatorname{LST} \tilde{U}_{2}^{*}\left(s_{1}\right)$. Now we are ready to give the main result of this section.

Theorem 2. In steady state, the joint workload distribution decomposes as an independent sum:

$$
\left(V^{(1)}, V^{(2)}, V^{(3)}\right) \stackrel{d}{=}\left(\tilde{V}^{(1), 1}, \tilde{V}^{(2), 1}, V^{(3)}\right)+\left(\tilde{V}^{(1), 2}, V^{(2), 2}, 0\right)+\left(V^{(1), 3}, 0,0\right) .
$$

The first term in the sum represents the steady-state distribution of the modified joint workload process obtained by removing the extra work in the first two queues at the end of a busy period in the third queue. The second term is the workload in the first two queues obtained by removing the extra work in the first queue at the end of a busy cycle in the second queue. Finally the third term represents the workload in the virtual $M / G / 1$ queue with input distributed as the extra work in queue 1, at the end of a busy period in queue 2.

Proof. Consider the modified work process that evolves in steady state as

$$
\left(\tilde{V}^{(1), 1}, \tilde{V}^{(2), 1}, V^{(3)}\right) \stackrel{d}{=}\left(\tilde{V}^{(1), 1}+B^{(1)}-A, \tilde{V}^{(2), 1}+B^{(2)}-A, V^{(3)}+B^{(3)}-A\right),
$$

if $A<V^{(3)}+B^{(3)}$; and $\left(\tilde{V}^{(1), 1}, \tilde{V}^{(2), 1}, V^{(3)}\right)=(0,0,0)$, else. 
By similar computations as the ones leading to Formula 11 , we obtain

$$
\tilde{\psi}\left(s_{1}, s_{2}, s_{3}\right)=\left(1-\rho_{3}\right) \frac{s_{1}+s_{2}+s_{3}-\lambda\left(1-U_{3}^{*}\left(s_{1}, s_{2}\right)\right)}{s_{1}+s_{2}+s_{3}-\lambda\left(1-\phi\left(s_{1}, s_{2}, s_{3}\right)\right)} .
$$

This is the first factor in (24). For the second one, consider the following modified virtual workload process that evolves in steady state as

$$
\left(\tilde{V}^{(1), 2}, V^{(2), 2}, 0\right) \stackrel{d}{=} \begin{cases}\left(\tilde{V}^{(1), 2}+U^{(1), 1}-A, V^{(2), 2}+U^{(2), 1}-A, 0\right), & \text { if } A<V^{(2), 2}+U^{(2), 1}, \\ (0,0,0), & \text { if } A \geq V^{(2), 2}+U^{(2), 1},\end{cases}
$$

with $\left(U^{(1), 1}, U^{(2), 1}\right)$ the extra work vector in the first 2 queues at the end of a busy period in queue 3. Here we remove the excess workload in the virtual queue 1 at the end of the busy period in the virtual queue 2 , which by Lemma 2 is the same as in the original system. In terms of LST's , this becomes

$$
\tilde{\psi}_{1}\left(s_{1}, s_{2}\right)=\frac{1-\rho_{1}}{1-\rho_{2}} \frac{s_{1}+s_{2}-\lambda\left(1-U_{2}^{*}\left(s_{1}\right)\right)}{s_{1}+s_{2}-\lambda\left(1-U_{3}^{*}\left(s_{1}, s_{2}\right)\right)} .
$$

Finally, the third factor in 24 is the Pollaczek-Khinchine representation of the steady-state workload in the $\mathrm{M} / \mathrm{G} / 1$ queue with service time distributed as the extra work in queue 1 at the end of a busy period in queue 2 . This ends the proof.

These considerations can be iterated now for the general $K$-dimensional system.

Corollary 1. The steady-state joint workload in the $K$ systems decomposes into the independent sum

$$
\begin{aligned}
\left(V^{(1)}, \ldots, V^{(K)}\right) \stackrel{d}{=}\left(\tilde{V}^{(1), 1}, \ldots, \tilde{V}^{(K-1), 1}, V^{(K)}\right)+\left(\tilde{V}^{(1), 2}, \ldots, \tilde{V}^{(K-2), 2}, V^{(K-1), 2}, 0\right) \\
\\
+\cdots+\left(\tilde{V}^{(1), K-1}, V^{(2), K-1}, 0, \ldots, 0\right)+\left(V^{(1), K}, 0, \ldots, 0\right),
\end{aligned}
$$

where the $j$ th term in the sum satisfies the identity in distribution $(j=2, \ldots, K)$ :

$$
\begin{aligned}
& \left(\tilde{V}^{(1), j}, \tilde{V}^{(2), j}, \ldots, \tilde{V}^{(K-j), j}, V^{(K-j+1), j}, 0, \ldots, 0\right) \stackrel{d}{=}\left(\tilde{V}^{(1), j}+U^{(1), j-1}-A,\right. \\
& \left.\quad \tilde{V}^{(2), j}+U^{(2), j-1}-A, \ldots, V^{(K-j+1), j}-B^{(K-j+1)}-A, 0, \ldots, 0\right), \\
& \quad \text { if } A \leq V^{(K-j+1), j}-B^{(K-j+1)},
\end{aligned}
$$

and $(0, \ldots, 0)$ else. $U^{(i), j}$ is the extra workload in queue $i$ at the end of a busy period in queue $(K-j+1)$, for $i>K-j+1$.

\section{The General TWO-DIMEnsional WORKLOAD/REINSURANCE PROBlem}

In this section we consider the general two-dimensional workload problem: pairs of customers arrive simultaneously at two parallel queues $Q_{1}$ and $Q_{2}$ according to a Poisson $(\lambda)$ process, the $n$th pair requiring service times $\left(B_{n}^{(1)}, B_{n}^{(2)}\right)$ with LST $\phi(s, t)$. We are interested in the steady-state workload vector $\left(V^{(1)}, V^{(2)}\right)$ with $\operatorname{LST} \psi(s, t)$. By the duality that is exposed in Section $2 \psi(s, t)$ also is the Laplace transform (w.r.t. $u_{1}$ and $u_{2}$ ) of the probability that both portfolios of an insurance 
company with simultaneous claims $\left(B_{n}^{(1)}, B_{n}^{(2)}\right)$, with initial capital $u_{1}$ and $u_{2}$, will survive.

In Section 3 we have determined $\psi(s, t)$ for the special case that $\mathbb{P}\left(B^{(1)} \geq B^{(2)}\right)=$ 1. We now show how the general case $-B_{n}^{(1)}$ and $B_{n}^{(2)}$ having an arbitrary joint distribution - has been solved in the literature (with the solution of that special case emerging as a degenerate solution). We shall successively discuss the contributions of Baccelli [4, De Klein [11] and Cohen [9, who have treated the two-dimensional workload problem with simultaneous arrivals in increasing generality. Starting point in all those three studies is the following functional equation for $\psi(s, t)$, which is derived by studying the 2-dimensional Markovian workload process during an infinitesimal amount of time $\Delta t$ :

$$
K(s, t) \psi(s, t)=t \psi_{1}(s)+s \psi_{2}(t), \mathcal{R} e s, t \geq 0 .
$$

Here the so-called kernel $K(s, t)$ is given by:

$$
K(s, t):=s+t-\lambda(1-\phi(s, t)),
$$

and

$$
\psi_{1}(s):=\mathbb{E}\left[\mathrm{e}^{-s V_{1}}\left(V_{2}=0\right)\right], \psi_{2}(t):=\mathbb{E}\left[\mathrm{e}^{-t V_{2}}\left(V_{1}=0\right)\right],
$$

with $(\cdot)$ denoting an indicator function.

Remark 3. In the special case of Section 3, with $\mathbb{P}\left(B^{(1)} \geq B^{(2)}\right)=1$, one has $\psi_{2}(t) \equiv \mathbb{P}\left(V_{1}=0\right)$, because $V_{2}$ cannot be positive when $V_{1}=0$. It then remains to find $\psi_{1}(s)$. This is done by observing (cf. the appendix) that, for all $s$ with $\mathcal{R} e s>0$, there is a unique zero $t(s)$ of the kernel, with $\mathcal{R} e t(s)>\mathcal{R} e(-s)$. This immediately yields that $\psi_{1}(s)=-\frac{s}{t(s)} \mathbb{P}\left(V_{1}=0\right)$, which is readily seen to be in agreement with (8).

Equation (5), which was obtained by studying the workloads at arrival epochs (i.e., the waiting times; by PASTA they have the same distribution as the steadystate workloads), looks slightly different from (25), but using (7) it is readily seen that they are equivalent.

Globally speaking, the essential steps in 4, 11, 9, are the following.

Step 1: find a suitable set of zeroes $(\hat{s}, \hat{t})$, with $\mathcal{R} e \hat{s} \geq 0$, $\mathcal{R} e \hat{t} \geq 0$, of the kernel $K(s, t)$, i.e., $K(\hat{s}, \hat{t})=0$. Because $\psi(s, t)$ is regular for all $(s, t)$ with $\mathcal{R} e s, t \geq 0$, one must have for all these zeroes:

$$
\hat{t} \psi_{1}(\hat{s})=-\hat{s} \psi_{2}(\hat{t}) .
$$

It is further observed that $\psi_{1}(s)$ is regular for $\mathcal{R} e s>0$, continuous for $\mathcal{R} e s \geq 0$, and that $\psi_{2}(t)$ is regular for $\mathcal{R} e t>0$, continuous for $\mathcal{R} e t \geq 0$.

Step 2: formulate a boundary value problem for $\psi_{1}(s)$ and $\psi_{2}(t)$. There are various types of boundary value problems, like the Riemann and the Wiener-Hopf boundary value problems. Typically, they ask to determine two functions $P_{1}(\cdot)$ and $P_{2}(\cdot)$, which satisfy a relation on a particular boundary $B$, while $P_{1}(\cdot)$ is regular in the interior $B^{+}$and $P_{2}(\cdot)$ is regular in the exterior $B^{-} . B$ could be the unit circle (Riemann boundary value problem), or the imaginary axis (Wiener-Hopf boundary value problem; $B^{+}$now is the left-half plane). We refer to Gakhov [16] and Mushkelishvili [19] for excellent expositions of such boundary value problems and their variants, like the boundary value problem with a shift. The latter occurs in 
the approach of De Klein [11, see below.

Step 3: solve the boundary value problem for $\psi_{1}(\cdot)$ and $\psi_{2}(\cdot)$ with boundary $B$. If $B$ is a smooth closed contour that is not a circle, the use of a conformal mapping from $B$ to the unit circle $C$ is required to arrive at a Riemann boundary value problem for the unit circle, the solution of which can be found in 16, 19. Thus one obtains $\psi_{1}(s)$ and $\psi_{2}(t)$ inside certain regions; subsequently, one may use analytic continuation to find them in $\mathcal{R} e s, t \geq 0$. Finally, $\psi(s, t)$ follows from 25).

Remark 4. Application of the boundary value method in queueing theory was pioneered by Fayolle and Iasnogorodski in [12. They used this method to analyze the joint queue length process in two coupled processors, viz., two $M / M / 1$ queues which operate at unit speeds when the other queue is not empty, but at different speeds when the other queue is empty. The method was subsequently developed in [10] for a large class of two-dimensional random walks; various queueing applications were also discussed in [10]. See [8] for a survey of the method in queueing theory, and see [13, 9] for two monographs which have further developed the theory of twodimensional random walks. Part IV of [9] explores the analysis of $N$-dimensional random walks with $N>2$. Results for $N>2$ are very limited, and it seems fair to conclude that the boundary value method is, apart from a few special cases, restricted to two-dimensional random walks.

Remark 5. We strongly believe that the boundary value method also has a large potential in the analysis of two-dimensional risk models. Due to the duality between the reinsurance model and the 2-queue model with simultaneous arrivals, the publications [4, 11, 9] are of immediate relevance to the reinsurance problem. These publications seem unknown in the insurance community (see, e.g., Chan et al. 17, who pose the two-dimensional risk problem and stop at Equation 25) (where [4, 11, 9 ] begin). They have remained largely unnoticed even in the queueing community, perhaps because of their complexity and because [4] and 11] did not appear in the open literature. For these reasons, we now successively expose the approaches in [4, [1] and 9 at some length.

The approach of Baccelli 4

Baccelli 4 restricts himself to the case of exchangeable $\left(B^{(1)}, B^{(2)}\right)$, i.e., $\mathbb{P}\left(B^{(1)}<\right.$ $\left.x, B^{(2)}<y\right)=\mathbb{P}\left(B^{(1)}<y, B^{(2)}<x\right)$, or equivalently, $\phi(s, t)=\phi(t, s)$. We briefly review the three steps mentioned above.

Step 1 in [4] is as follows. Consider zero pairs $(\hat{s}, \hat{t})=(g+i u, g-i u)$ of kernel $K(s, t)$, with $u \in \mathcal{R}$ and with $g=g(u)$ the unique zero in $\mathcal{R} e g \geq 0$ of

$$
2 g=\lambda(1-\phi(g+i u, g-i u)) .
$$

Using the exchangeability, it can be shown that this unique zero is real and nonnegative, while $g(-u)=g(u), u \in \mathcal{R}$.

Step 2. Consider the $\operatorname{arc} A=\{s: s=g(u)+i u, u \in R\}$, with $g(u)$ the zero defined above. This is a smooth arc, located in the right half-plane. Baccelli finds a conformal mapping $p(\cdot)$ of the interior $C^{+}$of the unit circle $C$ onto $A^{+}$, the 'interior' of $A$ located on the right of $A$, and a conformal mapping $q(\cdot)$ of $C^{-}$, the exterior of the unit circle, onto $A^{+}$; their limits on $C$ are denoted by $p^{+}(z)$ and $q^{-}(z)$, which are each other's complex conjugates because of the exchangeability. Noticing that $p^{+}(-1)=q^{-}(-1)=0$, he multiplies both sides of 28 with $1+z$. 
This yields (divide both sides of 28$)$ by $\hat{s} \hat{t})$ :

$$
(1+z) \frac{\psi_{1}\left(p^{+}(z)\right)}{p^{+}(z)}=-(1+z) \frac{\psi_{2}\left(q^{-}(z)\right)}{q^{-}(z)},|z|=1 .
$$

Because of the regularity properties of the conformal mappings and of $\psi_{1}(s)$ and $\psi_{2}(t), \mathcal{R} e s, t>0$, one now arrives at a simple boundary value problem: we have (29) for $|z|=1$, while the left-hand side of (29) is regular for $|z|<1$, and the right-hand side is regular for $|z|>1$.

Step 3. The solution of this problem immediately follows from Liouville's theorem, cf. 24] p. 85:

$$
\psi_{1}(p(z))=\frac{\gamma+\delta z}{1+z} p(z),|z|<1, \psi_{2}(q(z))=\frac{\gamma+\delta z}{1+z} q(z),|z|>1 .
$$

Baccelli [4] shows that $\gamma=-\delta$, and determines the remaining unknown constant $\delta$ by normalization. Having thus determined $\psi_{1}(s)$ for $s \in A^{+}$, he uses analytic continuation to obtain $\psi_{1}(s)$ in the whole right half-plane; similarly for $\psi_{2}(t)$. Finally, substitution in 25) determines $\psi(s, t)$.

The approach of De Klein 11]

De Klein [11, pp. 119-168, studies the general case of an arbitrary joint distribution of $B^{(1)}$ and $B^{(2)}$.

Step 1 in 11 is as follows. He considers the same zero pairs as Baccelli (also suggesting another set of zero pairs on p. 132). $g(u)$ is no longer necessarily real, but for all real $u$ there still is a unique zero $g(u)$.

Step 2. De Klein subsequently considers the simple, smooth arcs $A_{1}=\{s: s=$ $g(u)+i u, u \in R\}$ and $A_{2}=\{t: t=g(u)-i u, u \in R\}$ in the right half-plane. Notice that $A_{1}$ and $A_{2}$ are each other's complex conjugates in the exchangeable case of Baccelli, but not in De Klein's more general case. De Klein now uses the (unique) one-to-one mapping $t=\omega_{2}(s)$ from $A_{1}$ onto $A_{2}$ (with inverse $\omega_{1}(t)$ ) determined by the fact that, $\forall s \in A_{1},\left(s, \omega_{2}(s)\right)$ is a zero pair of the kernel. Similarly, $\forall t \in A_{2}$, $\left(\omega_{1}(t), t\right)$ is a zero pair. Hence the following must hold:

$$
\psi_{1}\left(\omega_{1}(t)\right)=-\frac{\omega_{1}(t)}{t} \psi_{2}(t), t \in A_{2} .
$$

In addition, one has the regularity properties of the functions $\psi_{1}(\cdot)$ and $\psi_{2}(\cdot)$ which were listed below (28). Determination of functions $\psi_{1}(\cdot)$ and $\psi_{2}(\cdot)$ with these regularity properties and satisfying (30) is a so-called shift problem, a boundary value problem with a shift (cf. Sections 17 and 18 of [16]).

Step 3. Gakhov [16] mentions two methods to solve such problems: (i) reduce the problem to a Fredholm integral equation of the second kind, and (ii) reduce the problem to an ordinary Riemann boundary value problem, by means of conformal mappings. De Klein [11] explores the first method in Section II.4.2 and the second in Section II.4.3. We concentrate on the first method. De Klein first translates the shift problem to one on a finite smooth closed contour, via the conformal mapping $\zeta(z)=\frac{1-z}{1+z}\left(\right.$ with inverse $\left.z(\zeta)=\frac{1-\zeta}{1+\zeta}\right)$ that maps $A_{i}$ onto smooth closed contours $T_{i}$, $i=1,2$; he then applies Gakhov's first method. He obtains the following Fredholm integral equation of the second kind for an unknown function $G_{1}(\cdot)$ - which up to 
a constant equals $\log \left\{\psi_{1}(z(\cdot))\right\}$ :

(31)

$G_{1}\left(p_{1}\right)=\frac{1}{2 \pi i} \int_{T_{1}} G_{1}\left(v_{1}\right)\left[\frac{1}{v_{1}-p_{1}}-\frac{\nu_{2}^{\prime}\left(v_{1}\right)}{\nu_{2}\left(v_{1}\right)-\nu_{2}\left(p_{1}\right)}-\frac{1}{v_{1}-c_{1}}\right] \mathrm{d} v_{1}+H_{1}\left(p_{1}\right), p_{1} \in T_{1}$,

with $H_{1}(\cdot)$ some known function, $c_{1}$ some point in the interior of $T_{1}$, and $\nu_{2}\left(v_{1}\right)$ $=\zeta\left(\omega_{2}\left(z\left(v_{1}\right)\right)\right), v_{1} \in T_{1}$. After having solved the integral equation (which can be done numerically in an efficient way, as shown by De Klein), one obtains $\psi_{1}(s)$ for $s \in T_{1}$, and then $\psi_{2}(t)$ for $t \in T_{2}$ via (28). The regularity of $\psi_{1}(s)$ in the interior $T_{1}^{+}$subsequently allows one to obtain $\psi_{1}(s), s \in T_{1}^{+}$, as a Cauchy integral; similarly for $\psi_{2}(t), t \in T_{2}^{+}$. By analytic continuation, $\psi_{1}(s)$ and $\psi_{2}(t)$ are then also uniquely determined in $\mathcal{R} e s \geq 0$ and $\mathcal{R} e t \geq 0$, respectively. Finally, $\psi(s, t)$ again follows from $(25)$.

De Klein also explores Gakhov's second method to treat the shift problem. However, this reduction to a Riemann boundary value problem requires a conformal mapping that itself must be determined by solving another Fredholm integral equation of the second kind. In Chapter II.6 he extensively investigates the numerical solution of both integral equations by means of the Nystrom or quadrature method. He obtains, a.o., accurate results for the mean sojourn time of a customer pair, viz., the time until both customers of a pair have left the system.

The approach of Cohen 9 ]

Cohen [9], Part III, considers a very general class of two-dimensional workload processes. Basically, he combines the model with simultaneous arrivals and the coupled processors model. The two servers have speeds $r_{1}$ and $r_{2}$ if they are both non-idle, and speeds $r^{(1)}$ and $r^{(2)}$ when the other server is idle. Furthermore, he also allows the possibility of different joint service requirement distributions if a customer pair arrives when at least one of the servers is idle. Finally, he explicitly allows single arrivals next to simultaneous arrivals (cf. also [6]). Much of Part III of [9] is devoted to a detailed study of the ergodicity conditions and of the so-called hitting point process and hitting point identity of the workload process, hitting point referring to the first entrance point of one of the axes.

In Chapter III.4 he determines the steady-state joint workload distribution for a variety of cases. For us, the most relevant cases are treated in Sections III.4.9 and III.4.10. Section III.4.9 treats the model of De Klein [11. The same zero pairs are used (Step 1), and the same smooth closed contours $T_{1}$ and $T_{2}$; Cohen subsequently uses Gakhov's second method to arrive at a Riemann boundary value problem (Step 2). That boundary value problem actually is so simple that it can be solved straightforwardly by applying Liouville's theorem, cf. Baccelli's method above (Step 3); however, a conformal mapping is required, which is obtained as the solution of another Fredholm integral equation of the second kind. A nice feature in Section III.4.9 is that $\psi_{1}(s)$ and $\psi_{2}(t)$, after normalization, are expressed as LST's of waiting time or workload distributions of special $M / G / 1$ queues (which are related to hitting points).

Section III.4.10 treats the model of De Klein with the additional feature that there is coupling of the servers, of a rather special form: $\frac{r_{1}}{r^{(1)}}+\frac{r_{2}}{r^{(2)}}=1$. This does not change the kernel $K(s, t)$ (which only refers to the interior of the state space, with both servers active), so the same zero pairs and contours can still be used. 
However, it does change the right-hand side of 25), and hence a slightly different Riemann boundary value problem must be solved.

Remark 6. It should be observed that Baccelli [4, De Klein [11] and Cohen 9] all also solve the more complicated transient problem, of determining the joint timedependent distribution of the two workloads.

\section{Conclusions And Future WOrK}

We have studied a multivariate queueing system, which is shown to correspond to a dual risk process with multiple lines of insurance that receive coupled claims. We find the LST of the multivariate workload distribution in the case in which the service requirements are ordered with probability one. Duality then yields the Laplace transform of the survival probabilities. For general service requirement (resp. claim size) vectors the workload (resp. ruin) problem can be solved in the two-dimensional case, by solving a Riemann boundary value problem. For dimension $K>2$, the problem seems analytically intractable in its full generality. That raises the need for approximations and asymptotics. It would in particular be interesting to obtain explicit multi-dimensional tail asymptotics of workloads and ruin probabilities, both for light-tailed and heavy-tailed service requirements (or claim sizes). Even for $K=2$ queues, this is already quite challenging. Moreover, a wide range of different cases must be studied, giving rise to quite different techniques and results. Therefore we intend to devote a separate study to tail asymptotics.

\section{APPENDIX}

Lemma 1 (Rouché zero). For every $s$ with $\mathcal{R} e s>0$ there exists a unique $t=t(s)$ with $\mathcal{R} e t(s)>\mathcal{R} e(-s)$, that satisfies the identity

$$
\lambda \phi(s, t)=\lambda-(s+t) .
$$

Moreover the function: $s \rightarrow t(s)$ is analytic in $\mathcal{R} e s>0$.

Proof. For fixed $s$ with $\mathcal{R} e s>0$, let $f(s+t):=\lambda-(s+t)$. Consider in the right half-plane the contour $\mathcal{C}$ made up from the semicircle with center at $-s$ and radius $R>2 \lambda$ together with the line segment $I:=\{-s+i w \mid w \in[-R, R]\}$. We show that on this contour $|\lambda \phi(s, t)|<|f(s+t)|$. We can bound $|\phi(s, t)|$ by

$$
\lambda|\phi(s, t)|=\lambda|\tilde{\phi}(s, s+t)| \leq \lambda \mathbb{E} e^{-\operatorname{Re} s\left(B^{(1)}-B^{(2)}\right)-\operatorname{Re}(t+s) B^{(2)}}<\lambda .
$$

This holds everywhere in the domain of $\phi(s, t)$ if $B^{(1)}-B^{(2)}$ has positive mass on $(0, \infty)$.

Now we bound $|f(s+t)|$. When $(s+t)$ is on the semicircle (i.e $|s+t|=R>2 \lambda$ ), apply the triangle inequality to the triangle with vertices at $0, \lambda, s+t$, to find $|\lambda-s-t|>\lambda$. When $(s+t) \in I$, by a similar argument we obtain $|\lambda-s-t| \geq \lambda$, with equality only when $s+t=0$. Hence on the contour $\mathcal{C},|f(s+t)| \geq \lambda$. We can now use Rouché's theorem to conclude that the equation $\lambda \phi(s, t)=\lambda-(s+t)$ has a unique solution $t(s)$ inside $\mathcal{C}$, because the polynomial $f(s+t)$ has only one zero inside $\mathcal{C}$, at $\lambda$. Letting $R \rightarrow \infty$, proves the assertion. 


\section{REFERENCES}

[1] Asmussen, S. And Albrecher, H. (2010). Ruin Probabilities. World Scientific Publ. Cy., Singapore.

[2] Avram, F., Palmowski, Z. and Pistorius, M. (2008). Exit problem of a two-dimensional risk process from the quadrant: Exact and asymptotic results. Annals of Applied Probability 18, 2421-2449.

[3] Avram, F., Palmowski, Z. and Pistorius, M. (2008). A two-dimensional ruin problem on the positive quadrant. Insurance: Mathematics and Economics 42, 227-234.

[4] BACCELli, F. (1985). Two parallel queues created by arrivals with two demands: The $M / G / 2$ symmetrical case. Technical report, INRIA-Rocquencourt.

[5] Baccelli, F., Makowski, A. And Shwartz, A. (1989). The fork-join queue and related systems with synchronization constraints: Stochastic ordering and computable bounds. Advances in Applied Probability 21, 629-660.

[6] Badescu, A., Cheung, E. and Rabehasaina, L. (2011). A two-dimensional risk model with proportional reinsurance. Journal of Applied Probability 48, 749-765.

[7] Chan, W.-S., Yang, H. and Zhang, L. (2003). Some results on ruin probabilities in a twodimensional risk model. Insurance: Mathematics and Economics 32, 345-358.

[8] Cohen, J. (1988). Boundary value problems in queueing theory. Queueing Systems 3, 97-128.

[9] Cohen, J. (1992). Analysis of Random Walks. IOS Press, Amsterdam, the Netherlands.

[10] Cohen, J. and Boxma, O. (1983). Boundary Value Problems in Queueing System Analysis. North-Holland Publ. Cy., Amsterdam.

[11] DE KLEIN, S. (1988). Fredholm Integral Equations in Queueing Analysis. PhD Thesis, University of Utrecht.

[12] Fayolle, G. and Iasnogorodski, R. (1979). Two coupled processors: The reduction to a Riemann-Hilbert problem. Z. Wahrsch. Verw. Gebiete 47, 325-351.

[13] Fayolle, G., Iasnogorodski, R. and Malyshev, V. (1999). Random Walks in the Quarter Plane. Springer, Berlin.

[14] Flatto, L. and Hahn, S. (1984). Two parallel queues created by arrivals with two demands. SIAM Journal of Applied Mathematics 44, 1041-1053.

[15] Frostig, E. (2004). Upper bounds on the expected time to ruin and on the expected recovery time. Annals of Applied Probability 36, 377-397.

[16] Gakhov, F. (1990). Boundary Value Problems. Pergamon Press, Oxford.

[17] Kella, O. (1993). Parallel and tandem fluid networks with dependent Lévy inputs. Annals of Applied Probability 3, 682-695.

[18] Löpker, A. AND Perry, D. (2010). The idle period of the finite $G / M / 1$ queue with an interpretation in risk theory. Queueing Systems 64, 395-407.

[19] Mushkelishvili, N. (2008). Singular Integral Equations 2nd ed. Dover Publications.

[20] Nelson, R. and Tantawi, A. (1987). Approximating task response times in fork/join queues. IBM T.J. Watson Research Center.

[21] Nelson, R. and Tantawi, A. (1988). Approximate analysis of fork/join synchronization in parallel queues. IEEE Transactions on Computers 37, 739-743.

[22] Rolski, T., Schmidli, H., Schmidt, V. And Teugels, J. (1999). Stochastic Processes for Insurance and Finance. Wiley Series in Probability and Statistics. Wiley, Chichester.

[23] Siegmund, D. (1976). The equivalence of absorbing and reflecting barrier problems for stochastically monotone markov processes. The Annals of Probability 4, pp. 914-924.

[24] Titchmarsh, E. (1968). The Theory of Functions 2nd corrected ed. Oxford University Press.

[25] Wright, P. (1992). Two parallel processors with coupled inputs. Annals of Applied Probability 24, 986-1007. 
Department of Mathematics and Computer Science, Eindhoven University of Technology, P.O. Box 513, 5600 MB Eindhoven, The Netherlands.

E-mail address: e.s.badila@tue.nl

E-mail address: boxma@win.tue.nl

E-mail address: resing@win.tue.nl

Korteweg de Vries Instituut voor Wiskunde, University of Amsterdam, P.O. Box 94248, 1090 GE Amsterdam, The Netherlands.

E-mail address: E.M.M.Winands@uva.nl 